\title{
Simple Gamma Rings With Involutions.
}

\author{
${ }^{1}$ A.C. Paul and ${ }^{2}$ Md. Sabur Uddin \\ ${ }^{I}$ Department of Mathematics University of Rajshahi, Rajshahi-6205 Bangladesh. \\ ${ }^{2}$ Associate Professor, Department of Mathematics, Carmichael College, Rangpur, Bangladesh
}

\begin{abstract}
Let $M$ be a simple gamma ring with an involution I. In this paper, we develop some characterizations of these gamma rings with involution. We also obtain some properties of Lie and Jordan ideals with involutions. Key words: Simple gamma rings, Involution, Symmetric elements, skew symmetric elements, Jordan ideals, Lie ideals.
\end{abstract}

2010 AMS Subject Classification : Primary 16 N 60, Secondary 16 W 25, 16 U 80.

\section{Introduction.}

The notion of a gamma ring was first introduced by N. Nobusawa [6] as a generalization of the concept of a classical ring. Barnes [1] generalized the concept of the Nobusawa's gamma ring which is known as a gamma ring and Nobusawa's gamma ring is known as $\Gamma_{\mathrm{N}}$-ring (i.e. gamma ring in the sense of Nobusawa) $\mathrm{L}$. Luh [5] worked on simple gamma rings and obtained some important properties. I. N. Herstein [3, 4] obtained various characterizations of simple rings with involution and also developed some structural results of Lie and Jordan rings. Paul and Sabur Uddin [7, 8] worked on Lie and Jordan structure in simple gamma rings and obtained some remarkable results.

In this paper, we introduce the concept of an involution of a $\Gamma$-ring. An example of the involution for a $\Gamma$-ring is given here. Some characterizations of simple $\Gamma$-rings are obtained by means of the involution. Also, we develop some properties of Lie and Jordan ideals with involutions.

\subsection{Definitions.}

\section{Preliminaries.}

Gamma Ring. [1] Let $M$ and $\Gamma$ be two additive abelian groups. Suppose that there is a mapping from $M \times \Gamma \times$ $\mathrm{M} \rightarrow \mathrm{M}$ (sending $(\mathrm{x}, \alpha, \mathrm{y})$ into $\mathrm{x} \alpha \mathrm{y})$ such that

i) $\quad(\mathrm{x}+\mathrm{y}) \alpha \mathrm{z}=\mathrm{x} \alpha \mathrm{z}+\mathrm{y} \alpha \mathrm{z}$

$x(\alpha+\beta) z=x \alpha z+x \beta z$

$x \alpha(y+z)=x \alpha y+x \alpha z$

ii) $\quad(x \alpha y) \beta z=x \alpha(y \beta z)$,

where $\mathrm{x}, \mathrm{y}, \mathrm{z} \in \mathrm{M}$ and $\alpha, \beta \in \Gamma$. Then $\mathrm{M}$ is called a $\Gamma$-ring.

Ideal of $\Gamma$-rings. A subset $A$ of the $\Gamma$-ring $\mathrm{M}$ is a left (right) ideal of $\mathrm{M}$ if $\mathrm{A}$ is an additive subgroup of $\mathrm{M}$ and $\mathrm{M} \Gamma \mathrm{A}=\{\mathrm{c} \alpha \mathrm{a} \mid \mathrm{c} \in \mathrm{M}, \alpha \in \Gamma, \mathrm{a} \in \mathrm{A}\}(\mathrm{A} \Gamma \mathrm{M})$ is contained in $\mathrm{A}$. If $\mathrm{A}$ is both a left and a right ideal of $\mathrm{M}$, then we say that $\mathrm{A}$ is an ideal or two sided ideal of $\mathrm{M}$.

If $A$ and $B$ are both left (respectively right or two sided) ideals of $M$, then $A+B=\{a+b \mid a \in A, b \in B\}$ is clearly a left (respectively right or two sided) ideal, called the sum of A and B. We can say every finite sum of left (respectively right or two sided) ideal of a $\Gamma$-ring is also a left (respectively right or two sided) ideal.

Nilpotent element. Let $M$ be a $\Gamma$-ring. An element $x$ of $M$ is called nilpotent if for some $\gamma \in \Gamma$, there exists a positive integer $\mathrm{n}=\mathrm{n}(\gamma)$ such that $(\mathrm{x} \gamma)^{\mathrm{n}} \mathrm{x}=(\mathrm{x} \gamma \mathrm{x} \gamma \ldots \gamma \mathrm{x} \gamma) \mathrm{x}=0$.

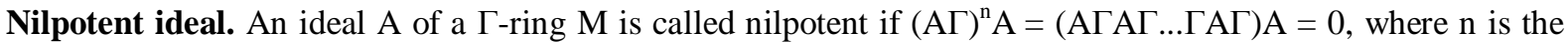
least positive integer.

Simple $\Gamma$-ring. $\mathrm{A} \Gamma$-ring $\mathrm{M}$ is called a simple $\Gamma$-ring if $\mathrm{M} \Gamma \mathrm{M} \neq 0$ and its ideals are $\{0\}$ and $\mathrm{M}$.

Centre of a $\Gamma$-ring. Let $\mathrm{M}$ be $\Gamma$-ring. The centre of $\mathrm{M}$, written as $\mathrm{Z}$ is the set of those elements in $\mathrm{M}$ that commute with every element in $\mathrm{M}$, that is, $\mathrm{Z}=\{\mathrm{m} \in \mathrm{M} \mid \mathrm{m} \gamma \mathrm{x}=\mathrm{x} \gamma \mathrm{m}$ for all $\mathrm{x} \in \mathrm{M}$ and $\gamma \in \Gamma\}$.

Jordan Structure. Let $\mathrm{M}$ be $\mathrm{a} \Gamma$-ring. The Jordan structure is defined by- $(\mathrm{x}, \mathrm{y})_{\alpha}=\mathrm{x} \alpha \mathrm{y}+\mathrm{y} \alpha \mathrm{x}$ for $\mathrm{x}, \mathrm{y} \in \mathrm{M}$ and all $\alpha \in \Gamma$. We say that a subset $A$ of $M$ is a Jordan sub- $\Gamma$-ring of $M$ if $A$ is an additive subgroup such that for a, $\mathrm{b} \in \mathrm{A}$ and $\alpha \in \Gamma, \mathrm{a} \alpha \mathrm{b}+\mathrm{b} \alpha \mathrm{a}$ must also be in $\mathrm{A}$. 
Jordan Ideal. Let $\mathrm{A}$ be a Jordan sub- $\Gamma$-ring of $\mathrm{M}$. The additive subgroup $\mathrm{U} \subset \mathrm{A}$ is to said to be a Jordan ideal of $A$ if whenever $\mathrm{u} \in \mathrm{U}, \mathrm{a} \in \mathrm{A}$, and $\alpha \in \Gamma$ then $(\mathrm{u}, \mathrm{a})_{\alpha}=\mathrm{u} \alpha \mathrm{a}+\mathrm{a} \alpha \mathrm{u}$ is in $\mathrm{U}$.

Lie Structure. Let $M$ be $a \Gamma$-ring. The Lie structure is defined by- $[x, y]_{\alpha}=x \alpha y-y \alpha x$ for $x, y \in M$ and for all $\alpha \in \Gamma$. We say that a subset $A$ of $M$ is a Lie sub- $\Gamma$-ring of $M$ if $A$ is an additive subgroup such that for $a, b \in A$ and $\alpha \in \Gamma$, a $\alpha$ b - boa must also be in A.

Lie Ideal. Let $\mathrm{A}$ be a Lie sub- $\Gamma$-ring of $\mathrm{M}$. The additive subgroup $\mathrm{U} \subset \mathrm{A}$ is said to be a Lie ideal of $\mathrm{A}$ if whenever $\mathrm{u} \in \mathrm{U}, \mathrm{a} \in \mathrm{A}, \alpha \in \Gamma$, then $[\mathrm{u}, \mathrm{a}]_{\alpha}=\mathrm{u} \alpha \mathrm{a}-\mathrm{a} \alpha \mathrm{u}$ is in $\mathrm{U}$. If $\mathrm{A}, \mathrm{B}$ are subsets of $\mathrm{M}$, then $[\mathrm{A}, \mathrm{B}]_{\Gamma}$ is the additive subgroup of $M$ generated by all $a \alpha b-b \alpha a$ with $a \in A, b \in B$ and $\alpha \in \Gamma$. If $M$ is non-commutative simple $\Gamma$-ring of characteristic $\neq 2$, then the sub- $\Gamma$-ring generated by $[\mathrm{M}, \mathrm{M}]_{\Gamma}$ in $\mathrm{M}$. If $\mathrm{U}$ is a Lie ideal of $\mathrm{M}$, let $\mathrm{T}(\mathrm{U})=\left\{\mathrm{x} \in \mathrm{M} \mid[\mathrm{x}, \mathrm{M}]_{\Gamma} \subset \mathrm{U}\right\}$.

We need the following theorems for obtaining our results which are appeared in [7, 8].

2.2 Theorem. Let $M$ be a simple $\Gamma$-ring of characteristic $\neq 2$. Then any Lie ideal of M which is also a sub- $\Gamma$ ring of $\mathrm{M}$ must either be $\mathrm{M}$ itself or contained in the centre of $\mathrm{M}$.

2.3 Theorem. Let $M$ be a $\Gamma$-ring and $Z$ is a centre of $M$. If $M$ is quadratic over $Z$, then $M$ is at most 4dimensional over $\mathrm{Z}$.

2.4 Theorem. If $\mathrm{M}$ is a simple $\Gamma$-ring and if $\mathrm{U}$ is a Lie ideal of $[\mathrm{M} . \mathrm{M}]_{\Gamma}$ then either $U \subset Z$ or $U \supset[\mathrm{M} . \mathrm{M}]_{\Gamma}$ except if $\mathrm{M}$ is of characteristic 2 and is 4-dimensional over $\mathrm{Z}$.

2.5 Theorem. If $\mathrm{M}$ is a simple non-commutative $\Gamma$-ring then the sub- $\Gamma$-ring generated by $[\mathrm{M}, \mathrm{M}]_{\Gamma}$ is $\mathrm{M}$.

2.6 Theorem. Let $M$ be a simple $\Gamma$-ring of characteristic $\neq 2$ and let $U$ be a Lie ideal of $M$. Then either $U \subset Z$ or $U \supset[\mathrm{M}, \mathrm{M}]_{\Gamma}$.

2.7 Theorem. If $M$ is a non-commutative simple $\Gamma$-ring of characteristic $\neq 2$, then the sub- $\Gamma$-ring generated by $[\mathrm{M}, \mathrm{M}]_{\Gamma}$ is $\mathrm{M}$.

2.8 Theorem. Let $\mathrm{M}$ be a $\Gamma$-ring and $0 \neq N$ a right ideal of $\mathrm{M}$. Suppose that, given $a \in N$ and $\gamma \in \Gamma,(a \gamma)^{n} a=0$ for a fixed integer $\mathrm{n}$; then $\mathrm{M}$ has a non-nilpotent ideal.

2.9 Theorem. Let $\mathrm{M}$ be a $\Gamma$-ring having no-non-zero nilpotent ideals in which $2 x=0$ implies that $x=0$. If $a \in \mathrm{M}$ commutes with all $a \alpha x-x \alpha a, x \in \mathrm{M}, \alpha \in \mathrm{M}$, then a is in the centre of M.

\section{Simple Gamma Rings with Involutions.}

3.1 Involution $\Gamma$-ring. Let $\mathrm{M}$ be a $\Gamma$-ring. A mapping $\mathrm{I}: \mathrm{M} \rightarrow \mathrm{M}$ is called an involution if

(i) $\mathrm{I}(a+b)=\mathrm{I}(a)+\mathrm{I}(b)$

(ii) $\mathrm{I}(a \alpha b)=\mathrm{I}(b) \alpha \mathrm{I}(a)$

(iii) $\mathrm{I}^{2}(a)=a$

for all $a, b \in M, \alpha \in \Gamma$.

If $\mathrm{I}(a)=a$, then a is called a symmetric element of $\mathrm{M}$ and if $\mathrm{I}(a)=-a$, then a called a skew symmetric element of $\mathrm{M}$.

3.2 Example. Let $\mathrm{R}$ be an associative ring with 1 having an involution *. Let

$$
\begin{aligned}
& \mathrm{M}=\mathrm{M}_{1.2}(\mathrm{R}) \text { and } \Gamma=\left\{\left(\begin{array}{c}
n_{1} \cdot 1 \\
n_{2} \cdot 1
\end{array}\right): n_{1}, n_{2} \in Z\right\} . \text { Then } \mathrm{M} \text { is a } \Gamma \text {-ring. Define } \mathrm{I}: \mathrm{M} \rightarrow \mathrm{M} \text { by } \\
& \mathrm{I}((\mathrm{a}, \mathrm{b}))=\left(\mathrm{a}^{*}, \mathrm{~b}^{*}\right) \text {. Then it is clear that } \mathrm{I} \text { is an involution on } \mathrm{M} \text {. }
\end{aligned}
$$

3.3 Theorem. Let $\mathrm{M}$ be a simple $\Gamma$-ring with an involution I on M. Define S, the set of all symmetric elements of $\mathrm{M}$ by $\mathrm{S}=\{x \in \mathrm{M} \mid \mathrm{I}(x)=x\}$ and $\mathrm{K}$, the set of all skew symmetric elements of $\mathrm{M}$ by

$\mathrm{K}=\{x \in \mathrm{M} \mid \mathrm{I}(x)=-x\}$. Then $\mathrm{S}$ and $\mathrm{K}$ are respectively Jordan sub- $\Gamma$-ring and Lie sub- $\Gamma$-ring of $\mathrm{M}$ and $\mathrm{M}$ $=S \oplus \mathrm{K}$. 
Proof. We have I ( 0$)=0$ then $0 \in S$. Let $a, b \in S$, then $\mathrm{I}(\mathrm{a}-\mathrm{b})=\mathrm{I}(\mathrm{a}) \mathrm{I}(\mathrm{b})=\mathrm{a}-\mathrm{b}$. So $a-b \in S$. Hence $\mathrm{S}$ in an additive subgroup of M. Let $\alpha \in \Gamma$, then $\mathrm{I}(a \alpha b+b \alpha a)=\mathrm{I}(a \alpha b)+\mathrm{I}(b \alpha a)=\mathrm{I}(b) \alpha \mathrm{I}(a)+\mathrm{I}(a) \alpha \mathrm{I}(b)=$ $b \alpha a+a \alpha b=a \alpha b+b \alpha a$. Thus $a \alpha b+b \alpha a \in S$. Hence $\mathrm{S}$ is a Jordan sub- $\Gamma$-ring of $\mathrm{M}$. We have $\mathrm{I}(0)=$ $0=-0$, so $0 \in \mathrm{K}$.

Let $a, b \in \mathrm{K}$, then $\mathrm{I}(\mathrm{a}-\mathrm{b})=\mathrm{I}(\mathrm{a})-\mathrm{I}(\mathrm{b})=-\mathrm{a}+\mathrm{b}=-(\mathrm{a}-\mathrm{b})$. Hence $a-b \in \mathrm{K}$. So $\mathrm{K}$ is an additive subgroup of $\mathrm{M}$. Let $\alpha \in \Gamma$, then

$$
\begin{aligned}
& \mathrm{I}(a \alpha b-b \alpha a)=\mathrm{I}(a \alpha b)-\mathrm{I}(b \alpha a) \\
& =\mathrm{I}(b) \alpha \mathrm{I}(a)-\mathrm{I}(a) \alpha \mathrm{I}(b) \\
& =(-b) \alpha(-a)-(-a) \alpha(-b) \\
& =b \alpha a-a \alpha b \\
& =-(a \alpha b-b \alpha a) . \text { Thus } a \alpha b-b \alpha a \in K .
\end{aligned}
$$

Hence $\mathrm{K}$ is a Lie sub- $\Gamma$-ring of $\mathrm{M}$. Since $2 \mathrm{M}$ is an ideal of $\mathrm{M}$ and $\mathrm{M}$ is simple, $2 \mathrm{M}=\mathrm{M}$. So for every $x \in \mathrm{M}, x / 2$ makes sense and so we can write $x=\frac{x+\mathrm{I}(x)}{2}+\frac{x-\mathrm{I}(x)}{2}$.

Now $\mathrm{I}\left(\frac{x+\mathrm{I}(x)}{2}\right)=\frac{1}{2} \mathrm{I}(x+\mathrm{I}(x))=\frac{1}{2}\left(\mathrm{I}(x)+\mathrm{I}^{2}(x)\right)=\frac{1}{2}(\mathrm{I}(x)+x)=\frac{1}{2}(x+\mathrm{I}(x))=\frac{x+\mathrm{I}(x)}{2}$.

Hence $\frac{x+\mathrm{I}(x)}{2} \in S$.

$\operatorname{Again} \mathrm{I}\left(\frac{x-\mathrm{I}(x)}{2}\right)=\frac{1}{2} \mathrm{I}(x-\mathrm{I}(x))=\frac{1}{2}\left(\mathrm{I}(x)-\mathrm{I}^{2}(x)\right)=\frac{1}{2}(\mathrm{I}(x)-x)=-\frac{1}{2}(x-\mathrm{I}(x))=-\frac{x-\mathrm{I}(x)}{2}$.

Hence $\frac{x-\mathrm{I}(x)}{2} \in \mathrm{K}$.

There fore $x=\frac{x+\mathrm{I}(x)}{2}+\frac{x-\mathrm{I}(x)}{2} \in S+K$. Hence $\mathrm{M}=\mathrm{S}+\mathrm{K}$.

Let $x \in S \cap K$, then $x \in S$ and K. So $\mathrm{I}(x)=x$ and $\mathrm{I}(x)=-x$. Therefore $x=-x$. This implies that $2 x=0$. So $x=0$. Thus $S \cap K=\{0\}$. Hence $\mathrm{M}=S \oplus K$.

Now we shall determine the nature of $\mathrm{S}$ as a Jordan $\Gamma$-ring and that of $\mathrm{K}$ as a Lie $\Gamma$-ring.

Also, if $s \in S$ and $k \in K$ then $s \alpha k-k \alpha s \in S$. In studying $\Gamma$-rings with involution I two cases immediately present themselves; these depend on the nature of the involution on a certain prescribed subset. The definition we are about to give should be made using the centroid rather than the centre, however in the material at hand it is the centre, even if it is 0 , that plays the crucial role.

Notation. If $A$ is a subset of $M$ then $\bar{A}$ will denote the sub- $\Gamma$-ring of $M$ generated by $A$.

3.4 Theorem. Let $M$ be a simple $\Gamma$-ring with involution $I$ of characteristic not 2 and let $=\{x \in \mathrm{M} \mid \mathrm{I}(x)=x\}$. Then $\bar{S}$, the sub- $\Gamma$-ring of $\mathrm{M}$ generated by $\mathrm{S}$ is $\mathrm{M}$ unless $\mathrm{M}$ is of dimension 4 or less (thus 4 or 1 ) over its centre.

Proof. We claim that $\bar{S}$ is a Lie ideal of M. To see this note first that trivially $[\bar{S}, S]_{\Gamma} \subset \bar{S}$. If $k \in K$ and $\bar{s} \in \bar{S}$ we want to show that $[\bar{s}, k]_{\alpha} \in \bar{S}, \alpha \in \Gamma$; to do so, since $\bar{S}$ is a sum of monomials from S, we need merely do it for monomials $\bar{s}=s_{1} \alpha s_{2} \alpha \ldots \ldots . \alpha s_{n}, s_{i} \in S$. But then $\left[s_{1} \alpha s_{2} \alpha \ldots \ldots \alpha s_{n}, k\right]_{\alpha}=\left[s_{1}, k\right]_{\alpha} \alpha s_{2} \alpha \ldots \ldots . \alpha s_{n}+\ldots \ldots+s_{1} \alpha \ldots \ldots \alpha s_{i-1} \alpha\left[s_{i}, k\right]_{\alpha} \alpha s_{i+1} \alpha \ldots \ldots . \alpha s_{n}+\ldots \ldots$. 
$+s_{1} \alpha s_{2} \alpha \ldots \ldots . \alpha s_{n-1} \alpha\left[s_{n}, k\right]_{\alpha}$ which certainly in $\bar{S}$. Thus

$[\bar{S}, \mathrm{M}]_{\Gamma}=[\bar{S}, S+K]_{\Gamma}=[\bar{S}, S]_{\Gamma}+[\bar{S}, K]_{\Gamma} \subset \bar{S}$ and so $\bar{S}$ is a Lie ideal of M. By definition it is a sub$\Gamma$-ring of M. There fore by Theorem 2.2 we conclude that either $\bar{S}=M$ or $\bar{S} \subset Z$.

We consider the second possibility, namely $\bar{S} \subset Z$. But then $S \subset Z$. Given $a \in \mathrm{M}, a=s+k, s \in S \subset Z, k \in K$ hence $a-s=k$. Then $(a-s) \gamma(a-s)=k \gamma k, \gamma \in \Gamma$. This implies that $\quad a \gamma a-a \gamma s-s \gamma a+s \gamma s=k \gamma k$. So $\quad a \gamma a-s \gamma a-s \gamma a+s \gamma s=k \gamma k$. Consequently $a \gamma a-2 s \gamma a+s \gamma s-k \gamma k=0$, which is to say, $\mathrm{M}$ is quadratic over $\mathrm{Z}$. By theorem 2.3, we get that $\mathrm{M}$ is at most 4-dimensional over $\mathrm{Z}$.

Related to this theorem is the following remark which holds for simple $\Gamma$-rings of any characteristic which have involutions.

3.5 Theorem. Let $\mathrm{M}$ be a simple $\Gamma$-ring with involution $\mathrm{I}$ whose centre $\mathrm{Z}=0$ or for which $\operatorname{dim} M / Z>4$. Then the only element commuting with $S=\{x \in \mathrm{M} \mid \mathrm{I}(x)=x\}$ lie in $Z$.

Proof. Let $a \in \mathrm{M}$ commute with all $s \in S$. If the characteristic of $\mathrm{M}$ is not 2, by theorem 3.4, $\bar{S}=\mathrm{M}$ hence $a \in \mathrm{Z}$ follows. Thus we may suppose that M is of characteristic 2 .

Let $\mathrm{T}=\{m \in \mathrm{M} \mid m \alpha s=s \alpha m, s \in S, \alpha \in \Gamma\} . \mathrm{T}$ is clearly a sub- $\Gamma$-ring of $\mathrm{M}$. Given $x \in \mathrm{M}, a \in \mathrm{T}$ then $a \alpha(x+\mathrm{I}(x))=(x+\mathrm{I}(x) \alpha a$. This implies that $a \alpha x+a \alpha \mathrm{I}(x)=x \alpha a+\mathrm{I}(x) \alpha a$. So that $a \alpha x+2 x \alpha a+a \alpha \mathrm{I}(x)=x \alpha a+I(x) \alpha a+2 a \alpha \mathrm{I}(x)$. Hence $a \alpha x+x \alpha a=a \alpha \mathrm{I}(x)+\mathrm{I}(x) \alpha a$.

We want to show that $\mathrm{T}$ is a Lie ideal of $\mathrm{M}$. Given $a \in \mathrm{T}, y \in \mathrm{M}, s \in S$ then

$$
\begin{aligned}
& (a \alpha y+y \alpha a) \alpha s=a \alpha y \alpha s+y \alpha a \alpha s \\
& \quad=a \alpha y \alpha s+y \alpha s \alpha a \\
& =a \alpha y \alpha s+2 a \alpha s \alpha \mathrm{I}(y)+y \alpha s \alpha a \quad \sin c e \quad 2 a \alpha s \alpha \mathrm{I}(y)=0 \\
& \quad=a \alpha y \alpha s+a \alpha s \alpha \mathrm{I}(y)+a \alpha s \alpha \mathrm{I}(y)+y \alpha s \alpha a
\end{aligned}
$$

as we have just shown. In other words, $\mathrm{T}$ is both a Lie ideal and sub- $\Gamma$-ring of $\mathrm{M}$. By our assumption on dim $M / Z$ we get from Theorems 2.4 and 2.5 that $T \subset Z$ or $T=\mathrm{M}$. If $\mathrm{T}=\mathrm{M}$ then $S \subset Z$ which we have seen forces $\operatorname{dim} R / Z \leq 4$. Thus $T \subset Z$, which is the assertion of the theorem.

We have already seen in Theorem 3.4 that $\bar{S}=\mathrm{M}$ for most simple $\Gamma$-rings. We now wish to establish its companion theorem namely, that $\bar{K}=\mathrm{M}$ in general. To do so we first show another construction, in most $\Gamma$ ring with involution I of a Lie ideal of the $\Gamma$-ring.

3.6 Definition. $\mathrm{K} \Gamma \mathrm{K}$ is the additive group generated by all $k_{1} \alpha k_{2}$ with $k_{1}, k_{2} \in K, \alpha \in \Gamma$.

3.7 Lemma. Let $\mathrm{M}$ be any $\Gamma$-ring with involution I such that $\mathrm{M}=\mathrm{S}+\mathrm{K}$. Then $\mathrm{K} \Gamma \mathrm{K}$ is a Lie ideal of $\mathrm{M}$.

Proof. Let $\quad k_{1}, k_{2} \in \mathrm{K} \quad k \in K$ and Then

$\left(k_{1} \alpha k_{2}\right) \alpha k-k \alpha\left(k_{1} \alpha k_{2}\right)=\left(k_{1} \alpha k-k \alpha k_{1}\right) \alpha k_{2}+k_{1} \alpha\left(k_{2} \alpha k-k \alpha k_{2}\right)$ 
$\in K \Gamma K$, so $[K \Gamma K, K]_{\Gamma} \subset K \Gamma K$. On the other hand, if $s \in S$ then $\left(k_{1} \alpha k_{2}\right) \alpha s-s \alpha\left(k_{1} \alpha k_{2}\right)=k_{1} \alpha\left(k_{2} \alpha s+s \alpha k_{2}\right)-\left(k_{1} \alpha s+s \alpha k_{1}\right) \alpha k_{2} \in K \Gamma K$.

Thus $[K \Gamma K, S]_{\Gamma} \subset K \Gamma K$. Now $[K \Gamma K, M]_{\Gamma}=[K \Gamma K, K+S]_{\Gamma}=[K \Gamma K, K]+[K \Gamma K, S]_{\Gamma} \subset K \Gamma K$. Hence $\mathrm{K} \Gamma \mathrm{K}$ is a Lie ideal of $\mathrm{M}$.

3.8 Theorem. If $M$ is a simple $\Gamma$-ring with involution I of characteristic not 2, then $\bar{K}=M$ provided dim $M / Z>4$.

Proof. Then conditions of Lemma 3.7 hold in $\mathrm{M}$ hence $\mathrm{K} \Gamma \mathrm{K}$, as a Lie ideal of $\mathrm{M}$. By theorem 2.6 must either $K \Gamma K \supset[M, M]_{\Gamma} \quad$ or $K \Gamma K \subset Z . \quad$ Now $\quad$ if $\quad K \Gamma K \supset[M, M]_{\Gamma} \quad$ then $\bar{K} \supset \overline{K \Gamma K} \supset[\overline{M, M}]_{\Gamma}=M$ by the theorem 2.7. Suppose then that $K \Gamma K \subset Z$.

If $a \in K$ is not invertible then since $a \Gamma K \subset Z$ and all the non-zero elements of $\mathrm{Z}$ are invertible we must have $a \Gamma K=0$. In particular, $a \alpha a=0, \alpha \in \Gamma$. If $a \in S$ then $a \alpha s+s \alpha a \in K$. Hence $0=a \alpha(a \alpha s+s \alpha a)=a \alpha a \alpha s+a \alpha s \alpha a=a \alpha s \alpha a$. There fore $\quad a \Gamma S \Gamma a=0$. Hence $a \Gamma \mathrm{M} \Gamma a=a \Gamma(S+K) \Gamma a=a \Gamma S \Gamma a+a \Gamma \mathrm{K} \Gamma a=0+0 \Gamma a=0$. Consequently $\mathrm{M} \Gamma$ a is a nilpotent left ideal and so $\mathrm{a}=0$. Thus $a \neq 0$ in $\mathrm{K}$ forces a to be invertible. If $\mathrm{b} \in \mathrm{K}$ and $a \alpha b \in Z$, then we get $b \in Z \Gamma a^{-1}=Z \Gamma a(\sin c e \quad a \alpha a=n \in Z)$. Thus $K \subset Z \Gamma a$.

If $\mathrm{s} \in \mathrm{S}$ commutes with a then $s \alpha a \in K \subset Z \Gamma a$ forcing $s \in Z$. Now if $s \in S$ then $s \alpha a+a \alpha s=t \alpha a, t \in Z$, in fact $t \in Z \cap S$. Thus $(s \alpha s-t \alpha s) \alpha a=a \alpha(s \alpha s-t \alpha s)$. But since $s \alpha s-t \alpha s \in S$ and commutes with a, so $s \alpha s-t \alpha s \in Z$. Given $x \in M, x=s+p \alpha a, s \in S, p \in Z$. Hence

$$
\begin{aligned}
x \alpha x & =(s+p \alpha a) \alpha(s+p \alpha a) \\
& =s \alpha s+s \alpha p \alpha a+p \alpha a \alpha s+(p \alpha a) \alpha(p \alpha a) \\
& =s \alpha s+p \alpha s \alpha a+p \alpha a \alpha s+p \alpha(a \alpha p) \alpha a \\
& =s \alpha s+p \alpha s \alpha a+p \alpha a \alpha s+p \alpha p \alpha a \alpha a \\
& =s \alpha s+p \alpha(s \alpha a+a \alpha s)+(p \alpha p) \alpha(a \alpha a) \\
& =s \alpha s+p \alpha t \alpha a+p \alpha p \alpha n .
\end{aligned}
$$

Now $x \alpha x-t \alpha x=s \alpha s+p \alpha t \alpha a+p \alpha p \alpha n-t \alpha x$

$$
\begin{aligned}
& =s \alpha s+p \alpha t \alpha a+p \alpha p \alpha n-t \alpha(s+p \alpha a) \\
& =s \alpha s+p \alpha t \alpha a+p \alpha p \alpha n-t \alpha s-t \alpha p \alpha a \\
& =s \alpha s+p \alpha t \alpha a+p \alpha p \alpha n-t \alpha s-p \alpha t \alpha a \\
& =s \alpha s-t \alpha s+p \alpha p \alpha n .
\end{aligned}
$$

Since as we have seen $s \alpha s-t \alpha s \in Z$, we must have $x \alpha x-t \alpha x \in Z$. In this way $\mathrm{M}$ has been shown to be quadratic over Z. By theorem 2.3, M must be at most 4-dimenional over $Z$. This proves the theorem.

We now prepare to study the Jordan structure of S. We begin with

3.9 Theorem. If $U \neq 0$ is a Jordan ideal of S then for $u \in U, m, s \in M$,

$$
m \alpha(u \alpha)^{3} u \alpha s+\mathrm{I}(s) \alpha(u \alpha)^{3} u \alpha \mathrm{I}(m) \in U .
$$

Proof. Then proof will consist of breaking $\mathrm{m}$ and $\mathrm{s}$ into their symmetric and skew symmetric parts and verifying that in these special instances the theorem holds. We do this in the sequence of three lemmas.

3.10 Lemma. If $x, y \in S$ and $u \in U$ then $x \alpha u \alpha y+y \alpha u \alpha x \in U, \alpha \in \Gamma$.

Proof. $2 x \alpha u \alpha x=\{x \alpha(x \alpha u+u \alpha x)+(x \alpha u+u \alpha x) \alpha x\}-\{x \alpha x \alpha u+u \alpha x \alpha x\}$.

Since

$x \alpha x \in S, x \alpha x \alpha u+u \alpha x \alpha x \in U$. Again since $x \alpha u+u \alpha x \in U$, 
so is $\{x \alpha(x \alpha u+u \alpha x)+(x \alpha u+u \alpha x) \alpha x\} \in U$. Thus $2 x \alpha u \alpha x \in U$. But we have $2 \mathrm{~S}=\mathrm{S}$, so we get $x \alpha u \alpha x \in U$. Now Linearizing on $\mathrm{x}$ we get $x \alpha u \alpha y \in U$. Similarly we get $y \alpha u \alpha x \in U$. Thus $x \alpha u \alpha y+y \alpha u \alpha x \in U$.

3.11 Lemma. If $s \in S, k \in K$ and $x \in U$ then $s \alpha u \alpha u \alpha k-k \alpha u \alpha u \alpha s \in U, \alpha \in \Gamma$.

Proof. Since $u \alpha k-k \alpha u$ is in S, $u \alpha u \alpha k-k \alpha u \alpha u=u \alpha(u \alpha k-k \alpha u)+(u \alpha k-k \alpha u) \alpha u$ is in U. Being a Jordan ideal of $\mathrm{S}, s \alpha(u \alpha u \alpha k-k \alpha u \alpha u)+(u \alpha u \alpha k-k \alpha u \alpha u) \alpha s \in U$. That is,

(1)

$s \alpha(u \alpha u \alpha k-k \alpha u \alpha u)+(u \alpha u \alpha k-k \alpha u \alpha u) \alpha s=s \alpha u \alpha u \alpha k-k \alpha u \alpha u \alpha s-s \alpha k \alpha u \alpha u+u \alpha u \alpha k \alpha s$ is in $\mathrm{U}$.

Consider

(2)

$k \alpha(u \alpha u \alpha s-s \alpha u \alpha u)+($ u $и \alpha s-s \alpha u \alpha u) \alpha k=k \alpha u \alpha u \alpha s-s \alpha u \alpha u \alpha k-k \alpha s \alpha u \alpha u+u \alpha u \alpha s \alpha k$.

Adding (1) and (2) the right sides add up to $u \alpha u \alpha(k \alpha s+s \alpha k)-(k \alpha s+s \alpha k) \alpha u \alpha u$ which, since $k \alpha s+s \alpha k \in K$, we have seen must be in $\mathrm{U}$. Therefore the sum of the left sides must be in $\mathrm{U}$; since the left side of (1) is already in $U$ we get that of (2) must also be in $U$. Now subtract (1) from (2); doing so we stay in $U$. The result on the right is $2($ s $\alpha u \alpha u \alpha k-k \alpha u \alpha u \alpha s) \in U$. Since $2 S=S$ this gives $s \alpha u \alpha u \alpha k-k \alpha u \alpha u \alpha s \in U$ for all $s \in S, k \in K, u \in U, \alpha \in \Gamma$, which is the desired result.

3.12 Lemma. If $a, b \in K$ and $u \in U$ then $a \alpha(u \alpha)^{3} u \alpha b+b \alpha(u \alpha)^{3} u \alpha a \in U, \alpha \in \Gamma$.

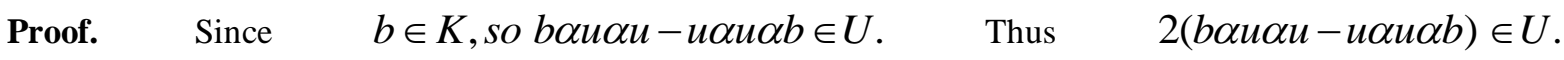

Since $2 K=K($ and so $4 K=K)$ this gives us $($ b $\alpha u \alpha u-u \alpha u \alpha b) \alpha(b \alpha u \alpha u-u \alpha u \alpha b) \in U$.

But expanding we have

$($ b $\alpha u \alpha u-u \alpha u \alpha b) \alpha(b \alpha u \alpha u-u \alpha u \alpha b)=(b \alpha u \alpha u) \alpha(b \alpha u \alpha u)-(b \alpha u \alpha u) \alpha(u \alpha u \alpha b)$

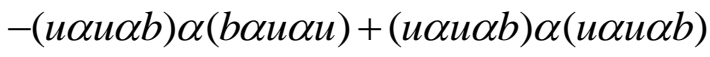

$$
\begin{aligned}
& =b \alpha u \alpha u \alpha b \alpha u \alpha u-b \alpha(u \alpha)^{3} u \alpha b-u \alpha u \alpha b \alpha b \alpha u \alpha u
\end{aligned}
$$

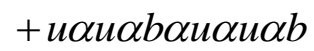

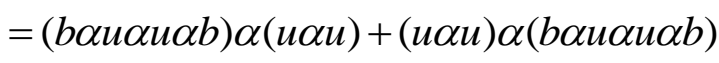

$$
\begin{aligned}
& -(u \alpha u) \alpha(b \alpha b) \alpha(u \alpha u)-b \alpha(u \alpha)^{3} u \alpha b .
\end{aligned}
$$

Now $\{(b \alpha u \alpha u \alpha b) \alpha(u \alpha u)+(u \alpha u) \alpha(b \alpha u \alpha u \alpha b)\}$ is in $\mathrm{U}$, since $b \alpha u \alpha u \alpha b \in S$ and $2 u \alpha u \in U$. By

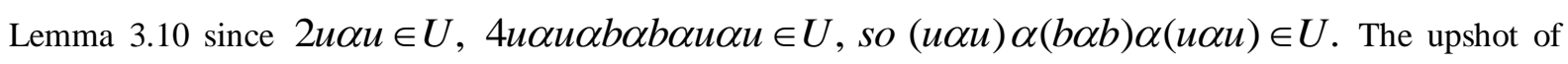
all this is that $b \alpha(u \alpha)^{3} u \alpha b \in U$. Linearizing on $\mathrm{b}$ we get $a \alpha(u \alpha)^{3} u \alpha b \in U$. Similarly we get $b \alpha(u \alpha)^{3} u \alpha a \in U$. Thus $a \alpha(u \alpha)^{3} u \alpha b+b \alpha(u \alpha)^{3} u \alpha a \in U$.

Proof of theorem 3.9. Given $u \in U, m, s \in M \quad$ then $m=m_{0}+m_{1}, s=s_{0}+s_{1} \quad$ with $m_{0}, s_{0}, \in S, m_{1}, s_{1} \in K$.

Thus

$$
m \alpha(u \alpha)^{3} u \alpha s+\mathrm{I}(s) \alpha(u \alpha)^{3} u \alpha \mathrm{I}(m)
$$

$=\left(m_{0}+m_{1}\right) \alpha(u \alpha)^{3} u \alpha\left(s_{0}+s_{1}\right)+\mathrm{I}\left(s_{0}+s_{1}\right) \alpha(u \alpha)^{3} u a \mathrm{I}\left(m_{0}+m_{1}\right)$

$=\left(m_{0}+m_{1}\right) \alpha(u \alpha)^{3} u \alpha\left(s_{0}+s_{1}\right)+\left(\mathrm{I}\left(s_{0}\right)+\mathrm{I}\left(s_{1}\right)\right) \alpha(u \alpha)^{3} u \alpha\left(\mathrm{I}\left(m_{0}\right)+\mathrm{I}\left(m_{1}\right)\right)$

$=\left(m_{0}+m_{1}\right) \alpha(u \alpha)^{3} u \alpha\left(s_{0}+s_{1}\right)+\left(s_{0}-s_{1}\right) \alpha(u \alpha)^{3} u \alpha\left(m_{0}-m_{1}\right)$

$=m_{0} \alpha(u \alpha)^{3} u \alpha s_{0}+s_{0} \alpha(u \alpha)^{3} u \alpha m_{0}+\left(m_{0} \alpha(u \alpha)^{3} u \alpha s_{1}-s_{1} \alpha(u \alpha)^{3} u \alpha m_{0}\right)$

$+\left(m_{1}(u \alpha)^{3} u \alpha s_{0}-s_{0} \alpha(m \alpha)^{3} u \alpha m_{1}\right)+\left(m_{1}(u \alpha)^{3} u \alpha s_{1}+s_{1} \alpha(u \alpha)^{3} u \alpha m_{1}\right)$.

Since $4(u \alpha)^{3} u \in U$ and since we have seen the factor 4 can be eliminated we obtain the desired theorem as a combination of the three Lammas 3.10, 3.11 and 3.12. 
We are in a position to prove the basic

3.13 Theorem. The only Jordan ideals of $S$ are 0 and $S$ that is, $S$ is a simple Jordan $\Gamma$-ring.

Proof. Let $U \neq 0$ be a Jordan ideal of S. If $u \in U$ then we have seen that $m \alpha(u \alpha)^{3} u \alpha t+\mathrm{I}(t) \alpha(u \alpha)^{3} u \alpha \mathrm{I}(m) \in U$ for all $\mathrm{m}, \mathrm{t} \in M$. If $(u \alpha)^{3} u \neq 0$ then $\mathrm{M} \Gamma(u \alpha)^{3} u \Gamma M=M$ and so, given $x \in M$ then $x=\sum m_{i} \alpha(u \alpha)^{3} u \alpha t_{i}$. But then $\mathrm{I}(x)=\sum \mathrm{I}\left(t_{i}\right) \alpha(u \alpha)^{3} u \alpha \mathrm{I}\left(m_{i}\right)$. Hence $x+\mathrm{I}(x)=\sum\left(m_{i} \alpha(u \alpha) u \alpha t_{i}+\mathrm{I}\left(t_{i}\right) \alpha(u \alpha)^{3} u \alpha \mathrm{I}\left(m_{i}\right)\right)$ is in $\mathrm{U}$. Since $x+\mathrm{I}(x)$ covers $\mathrm{S}$ as $x$ runs over $\mathrm{M}$ we get that $\mathrm{U}=\mathrm{S}$. Thus if $U \neq S$ we must assume that $(u \alpha)^{3} u=0$ for all $u \in U$. Given $u \in U, m=m_{0}+m_{1}, m_{0} \in S, m_{1} \in K$ we have

$$
\begin{aligned}
2\{u \alpha u \alpha m+\mathrm{I}(m) \alpha u \alpha u\} & =2\left\{u \alpha u \alpha\left(m_{0}+m_{1}\right)+\mathrm{I}\left(m_{0}+m_{1}\right) \alpha u \alpha u\right\} \\
& =2 u \alpha u \alpha m_{0}+2 u \alpha u \alpha m_{1}+2\left(\mathrm{I}\left(m_{0}\right)+\mathrm{I}\left(m_{1}\right)\right) \alpha u \alpha u \\
& =2 u \alpha u \alpha m_{0}+2 u \alpha u \alpha m_{1}+2\left(m_{0}-m_{1}\right) \alpha u \alpha u \\
& =2 u \alpha u \alpha m_{0}+2 u \alpha u \alpha m_{1}+2 m_{0} \alpha u \alpha u-2 m_{1} \alpha u \alpha u \\
& =2\left(u \alpha u \alpha m_{0}+m_{0} \alpha u \alpha u\right)+2\left(u \alpha u \alpha m_{1}-m_{1} \alpha u \alpha u\right) \text { is in U. }
\end{aligned}
$$

Since $\quad(u \alpha)^{3} u=0,4\{(u \alpha u \alpha m+\mathrm{I}(m) \alpha u \alpha u) \alpha\}^{3}(u \alpha u \alpha m+\mathrm{I}(m) \alpha u \alpha u)=0 . \quad$ We get

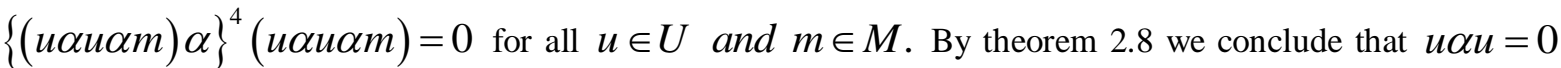
for all $u \in U$. Linearizing we get that $u \alpha v+v \alpha u=0$ for all $u, v \in U$. Given $s \in S, v=u \alpha s+s \alpha u \in U$. Hence

$$
0=u \alpha v+v \alpha u=u \alpha(u \alpha s+s \alpha u)+(u \alpha s+s \alpha u) \alpha u=u \alpha u \alpha s+u \alpha s \alpha u
$$
$+u \alpha s \alpha u+s \alpha u \alpha u=2 u \alpha s \alpha u+u \alpha u \alpha s+s \alpha u \alpha u=2 u \alpha s \alpha u$, since $u \alpha u=0$. Thus $u \alpha s \alpha u=0$ for all $u \in U$ and $s \in S$. Given $k \in K$ then $k \alpha u \alpha k \in S$. Hence $u \alpha k \alpha u \alpha k \alpha u=0$. For any $m \in M, m=m_{0}+m_{1}$ with $m_{0} \in S, m_{1} \in K$, then

$$
\begin{aligned}
& \text { иктаи атои }=и \alpha\left(m_{0}+m_{1}\right) \alpha и \alpha\left(m_{0}+m_{1}\right) \alpha u \\
& =\left(u \alpha m_{0} \alpha u+u \alpha m_{1} \alpha u\right) \alpha\left(m_{0}+m_{1}\right) \alpha u \\
& =\left(u \alpha m_{0} \alpha u+u \alpha m_{1} \alpha u\right) \alpha\left(m_{0} \alpha u+m_{1} \alpha u\right) \\
& =u \alpha m_{0} \alpha u \alpha m_{0} \alpha u+u \alpha m_{0} \alpha u \alpha m_{1} \alpha u+u \alpha m_{1} \alpha u \alpha m_{0} \alpha u+u \alpha m_{1} \alpha u \alpha m_{1} \alpha u \\
& =0 \text {. }
\end{aligned}
$$

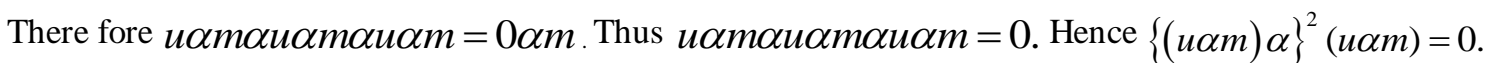

By Theorem 2.8, we conclude that $\mathrm{u}=0$. We have prove that $\mathrm{U}=0$ or $\mathrm{U}=\mathrm{S}$. Hence the theorem is proved. Having determined the Jordan structure of $\mathrm{S}$ we now want to determine the Lie structure of $\mathrm{K}$. We begin with the very easy

3.14 Lemma. If $\mathrm{U}$ is a Lie ideal of $\mathrm{K}$ and if $u \in U, s \in S$ then $(u \alpha u) \alpha s-s \alpha(u \alpha u) \in U, \alpha \in \Gamma$.

Proof. To see the result merely note that $u \alpha s+s \alpha u \in K$ and $(u \alpha u) \alpha s-s \alpha(u \alpha u)=u \alpha(u \alpha s+s \alpha u)-(u \alpha s+s \alpha u) \alpha u \in U$.

3.15 Definition. If $\mathrm{U}$ is a Lie ideal of $\mathrm{K}$ then $\mathrm{T}(U)=\left\{x \in K \mid[x, \mathrm{~K}]_{\Gamma} \subset U\right\}$.

Clearly $\mathrm{T}(\mathrm{U})$ is a Lie ideal of $\mathrm{K}$ and contains $\mathrm{U}$. We want a closer tie-in between $\mathrm{U}$ and $\mathrm{T}(\mathrm{U})$.

3.16 Lemma. If $\mathrm{U}$ is a Lie ideal of $\mathrm{K}$ then $u, v, w \in U$ implies $u \alpha v \alpha u \in T(U)$ and $u \alpha v \alpha w+w \alpha v \alpha u \in \mathrm{T}(U), \alpha \in \Gamma$.

Proof. Consider $[u \alpha v \alpha u, K]_{\Gamma} ;$ for $k \in K$ $u \alpha v \alpha u \alpha k-k \alpha u \alpha v \alpha u=u \alpha(v \alpha u \alpha k+k \alpha u \alpha v)-(v \alpha u \alpha k+k \alpha u \alpha v) \alpha u+v \alpha u \alpha k \alpha u-u \alpha k \alpha u \alpha v$

$$
\begin{aligned}
= & u \alpha\{v \alpha u \alpha k-\mathrm{I}(v \alpha u \alpha k)\}-\{(v \alpha u \alpha k-\mathrm{I}(v \alpha u \alpha k)) \alpha u\} \\
& +\{v \alpha(u \alpha k \alpha u)-(u \alpha k \alpha u) \alpha v\} .
\end{aligned}
$$




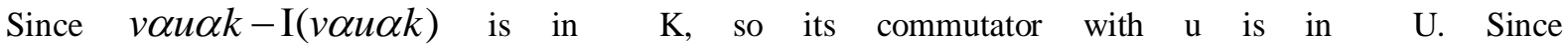
$u \alpha k \alpha u \in K,\{v \alpha(u \alpha k \alpha u)-(u \alpha k \alpha u) \alpha v\} \in U$. In all we have shown that $[u \alpha v \alpha u, K]_{\Gamma} \subset U$ and so $u \alpha v \alpha u \in \mathrm{T}(U)$. Linearizing on $\mathrm{U}$ we obtain $u \alpha v \alpha w+w \alpha v \alpha u \in \mathrm{T}(U)$.

We proceed to prove

3.17 Theorem. If $\mathrm{U}$ is a Lie ideal of $\mathrm{K}$ then for all $u, v \in U,(u \alpha u \alpha v-v \alpha u \alpha u) \alpha m-\mathrm{I}(m) \alpha(u \alpha u \alpha v-v \alpha u \alpha u) \in T(U)$ for all $m \in M, \alpha \in \Gamma$.

Proof. We write $m=s+k$ with $s \in S, k \in K$. Then

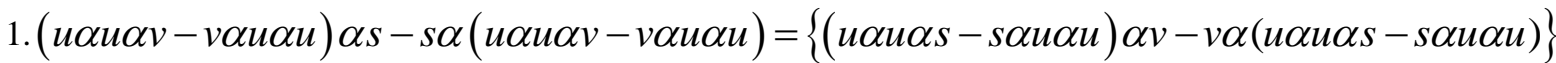

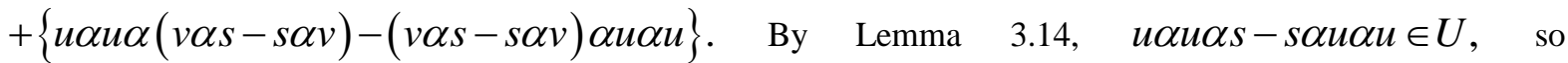
$\{(u \alpha u \alpha s-s \alpha u \alpha u) \alpha v-v \alpha(u \alpha u \alpha s-s \alpha u \alpha u)\}$ is in U. Also, since $v \alpha s-s \alpha v \in S$, by Lemma 3.14 again $\{u \alpha u \alpha(v \alpha s-s \alpha v)-(v \alpha s-s \alpha v) \alpha u \alpha u\}$ is in $\mathrm{U}$. Being in $\mathrm{U}$ these are certainly in $\mathrm{T}(\mathrm{U})$. Hence $(u \alpha u \alpha v-v \alpha u \alpha u) \alpha s-s \alpha(u \alpha u \alpha v-v \alpha u \alpha u)$ is in $\mathrm{T}(\mathrm{U})$.

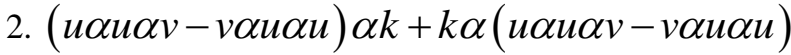

$=u \alpha u \alpha(v \alpha k+k \alpha v)-(v \alpha k+k \alpha v) \alpha u \alpha u\}+v \alpha(k \alpha u \alpha u-u \alpha u \alpha k)+(k \alpha u \alpha u-u \alpha u \alpha k) \alpha v$

$\equiv v \alpha(k \alpha u \alpha u-u \alpha u \alpha k)+(k \alpha u \alpha u-u \alpha u \alpha k) \alpha v(\bmod U)(b y L e m m a 3.14, \sin c e v \alpha k+k \alpha v \in S)$

$\equiv v \alpha((k \alpha u-u \alpha k) \alpha u+u \alpha(k \alpha u-u \alpha k))+((k \alpha u-u \alpha k) \alpha u+u \alpha(k \alpha u-u \alpha k)) \alpha v(\bmod U)$

$\equiv v \alpha\{(k \alpha u-u \alpha k) \alpha u-u \alpha(k \alpha u-u \alpha k)\}-\{(k \alpha u-u \alpha k) \alpha u-u \alpha(k \alpha u-u \alpha k)\} \alpha v+$

$2(v \alpha u \alpha(k \alpha u-u \alpha k)+(k \alpha u-u \alpha k) \alpha u \alpha v)(\bmod U)$

$\equiv 2(v \alpha u \alpha(k \alpha u-u \alpha k)+(k \alpha u-u \alpha k) \alpha u \alpha v)(\bmod U)$.

But by Lemma 3.14, since $k \alpha u-u \alpha k \in U, v \alpha u \alpha(u \alpha k-k \alpha u)+(u \alpha k-k \alpha u) \alpha u \alpha v$ is in $\mathrm{T}(\mathrm{U})$. Then upshot of all this is that $(u \alpha u \alpha v-v \alpha u \alpha u) \alpha k+k \alpha(u \alpha u \alpha v-v \alpha u \alpha u) \in \mathrm{T}(U)$.

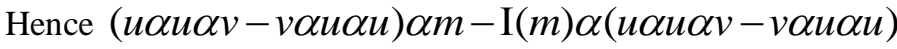

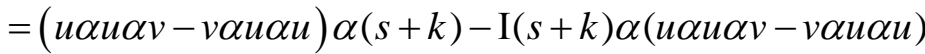

$=(u \alpha u \alpha v-v \alpha u \alpha u) \alpha(s+k)-(s-k) \alpha(u \alpha u \alpha v-v \alpha u \alpha u)$

$=\{(u \alpha u \alpha v-v \alpha u \alpha u) \alpha s-s \alpha(u \alpha u \alpha v-v \alpha u \alpha u)\}+\{(u \alpha u \alpha v-v \alpha u \alpha u) \alpha k+k \alpha(u \alpha u \alpha v-v \alpha u \alpha u)\}$ is in $\mathrm{T}(U)$.

3.18 Theorem. If $\mathrm{M}$ is simple and $\operatorname{dim} M / Z>4$ and if $\mathrm{U}$ is a Lie ideal of $\mathrm{K}$ then either $U \supset[K, K]_{\Gamma}$ or $u \alpha u \alpha v=v \alpha u \alpha u$ for all $u, v \in U$.

Proof. Let $a=u \alpha u \alpha v-v \alpha u \alpha u$, where $u, v \in U$. By theorem 3.17, $a \alpha m-\mathrm{I}(m) \alpha a \in \mathrm{T}(U)$ for all $m \in M, \alpha \in \Gamma$. If $k_{1} \in K$ then $b=(a \alpha m-\mathrm{I}(m) \alpha a) \alpha k_{1}-k_{1} \alpha(a \alpha m-\mathrm{I}(m) \alpha a) \in U \subseteq \mathrm{T}(U)$. Since $b=a \alpha\left(m \alpha k_{1}\right)-\mathrm{I}\left(m \alpha k_{1}\right) \alpha a-\mathrm{I}(m) \alpha a \alpha k_{1}-k_{1} \alpha a \alpha m$, so $\mathrm{I}(m) \alpha a \alpha k_{1}+k_{1} \alpha a \alpha m \in \mathrm{T}(U)$ for all $m \in M, k_{1} \in K$. We continue in this vein, let $k_{2} \in K$. Then

$\left(\mathrm{I}(m) \alpha a \alpha k_{1}+k_{1} \alpha a \alpha m\right) \alpha k_{2}-k_{2} \alpha\left(\mathrm{I}(m) \alpha a \alpha k_{1}+k_{1} \alpha a \alpha m\right) \in \mathrm{T} \subseteq \mathrm{T}(U)$

Hence $\mathrm{I}(m) \alpha a \alpha k_{1} \alpha k_{2}-\mathrm{I}\left(\mathrm{I}(m) \alpha a \alpha k_{1} \alpha k_{2}\right)+k_{1} \alpha a \alpha\left(m \alpha k_{2}\right)+\mathrm{I}\left(m \alpha k_{2}\right) \alpha a \alpha k_{1} \in \mathrm{T}(U)$.

Since $\quad k_{1} \alpha a \alpha\left(m \alpha k_{2}\right)+\mathrm{I}\left(m \alpha k_{2}\right) \alpha a \alpha k_{1} \in \mathrm{T}(U), \quad$ we obtain $\mathrm{I}(m) \alpha a \alpha k_{1} \alpha k_{2}-\mathrm{I}\left(\mathrm{I}(m) \alpha a \alpha k_{1} \alpha k_{2}\right) \in \mathrm{T}(U)$. Continuing we get by induction that for all $\bar{k} \in K$, $\mathrm{I}(m) \alpha a \alpha \bar{k}-\mathrm{I}(\mathrm{I}(m) \alpha a \alpha \bar{k}) \in \mathrm{T}(U)$. Since $\operatorname{dim} M / Z>4$, by theorem 3.8, $\bar{K}=M$. Then 
$m \alpha a \alpha t-\mathrm{I}(m \alpha a \alpha t) \in \mathrm{T}(U)$ for all $m, t \in \mathrm{M}$. Now $\mathrm{M} \Gamma \Gamma \Gamma \mathrm{M}$ is an ideal of $\mathrm{M}$, if $a \neq 0$, then $\mathrm{M} \Gamma$ a $\Gamma \mathrm{M}=\mathrm{M}$ But for any $x \in \mathrm{M}, x=\sum m_{i} \alpha a \alpha t_{i}, \quad$ so $\mathrm{I}(x)=\mathrm{I}\left(\sum m_{i} \alpha a \alpha t_{i}\right)=\sum \mathrm{I}\left(m_{i} \alpha a \alpha t_{i}\right)$ $=\sum \mathrm{I}\left(t_{i}\right) \alpha \mathrm{I}(a) \alpha \mathrm{I}\left(m_{i}\right)=\sum \mathrm{I}\left(t_{i}\right) \alpha a \alpha \mathrm{I}\left(m_{i}\right), \sin c e \mathrm{I}(a)=a$.

Hence $x-\mathrm{I}(x)=\sum m_{i} \alpha a \alpha t_{i}-\sum \mathrm{I}\left(m_{i} \alpha a \alpha t_{i}\right)=\sum\left(m_{i} \alpha a \alpha t_{i}-\mathrm{I}\left(m_{i} \alpha a \alpha t_{i}\right)\right) \in \mathrm{T}(U)$.

Since $x-\mathrm{I}(x)$ sweeps out $\mathrm{K}$ and we have that if $a \neq 0$ then $\mathrm{T}(U) \supset K$. From the definition of $\mathrm{T}(\mathrm{U})$ this says that $U \supset[K, K]_{\Gamma}$.

3.19 Theorem. If $\mathrm{M}$ is simple, $\operatorname{dim} M / Z>4$ and if $\mathrm{U}$ is a Lie ideal of $\mathrm{K}$ such that $[K, K]_{\Gamma} \not \subset U$ then given $u \in U, u \alpha u \in Z, \alpha \in \Gamma$.

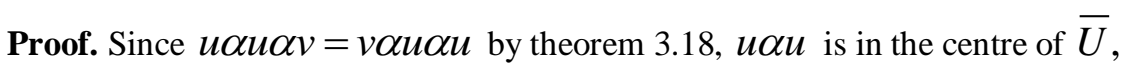

the sub- $\Gamma$-ring generated by U. However $u \alpha u \alpha s-s \alpha u \alpha u \in U$ for $s \in S$ and $u \alpha u \alpha k-k \alpha u \alpha u=u \alpha(u \alpha k-k \alpha u)+(u \alpha k-k \alpha u) \alpha u \in \bar{U}$ for $k \in S$, thus $u \alpha u$ commutes with all

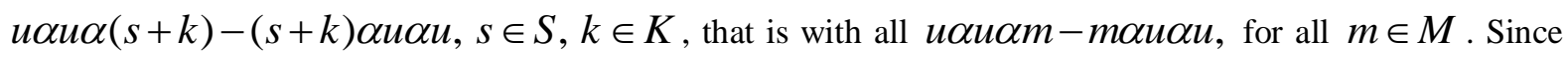
the characteristic is not 2 , by theorem 2.9 that this forces $u \alpha u$ to be in $\mathrm{Z}$.

3.20 Corollary. If $[K, K]_{\Gamma} \not \subset U$, then $u \alpha v+v \alpha u \in Z$ for all $u, v \in U$ and $\alpha \in \Gamma$.

3.21 Theorem. If $\mathrm{M}$ is simple, $\operatorname{dim} M / Z>4$ and $\mathrm{U}$ is a Lie ideal of $\mathrm{K}$ such that $u \alpha u \in U, \alpha \in \Gamma$ implies $u \alpha u=0$, then $\mathrm{U}=0$.

Proof. On linearizing $u \alpha u=0$ we get $u \alpha v+v \alpha u=0$ for all $u, v \in U$. Thus $u \alpha v=-v \alpha u$. So $u \alpha u \alpha v=-v \alpha u \alpha u=0 . \quad$ Given $\quad u \in U, k \in K \quad$ then $2 u \alpha k \alpha u=u \alpha k \alpha u-k \alpha u \alpha u-u \alpha u \alpha k+u \alpha k \alpha u=(u \alpha k-k \alpha u) \alpha u-u \alpha(u \alpha k-k \alpha u)(\sin c e u \alpha u=0)$. Hence $u \alpha k \alpha u \in U$. But then $v \alpha u \alpha k \alpha u \alpha v=0$. Since $u \alpha v=-v \alpha u$, we arrive at $u \alpha v \Gamma K \Gamma u \alpha v=0$. Now $\mathrm{I}(u \alpha v)=\mathrm{I}(v) \alpha \mathrm{I}(u)=v \alpha u=-u \alpha v$, that is, $u \alpha v \in \mathrm{K}$, thus for $s \in S, s \alpha u \alpha v \alpha s \in \mathrm{K}$ and so $u \alpha v \alpha s \alpha u \alpha v \alpha s \alpha u \alpha v \in u \alpha v \Gamma \mathrm{K} \Gamma u \alpha v=0$. Given $m \in S, \quad m=s+k, s \in S, k \in K, \quad$ whence $u \alpha v \alpha m \alpha u \alpha v \alpha m \alpha u \alpha v=0$. The right ideal $u \alpha \nu \Gamma \mathrm{M}$ is such that every element in it has cube 0 . By theorem 2.8 this forces $u \alpha v=0$ for all $u, v \in U$. But then for $k \in \mathrm{K}, u \alpha(u \alpha k-k \alpha u)=0$, leaving us with $u \Gamma \mathrm{K} \Gamma u=0$. As above we then get $u \alpha s \alpha u \alpha s \alpha u=0$ for $s \in S$ and so $u \Gamma \mathrm{M}$ is a nil right ideal, where every element has cube 0 . The outcome of this is that $u=0$ that is, $U=0$.

Combining theorems 3.19 and 3.21 we have

3.22 Theorem. If $M$ is simple and $Z=0$ then any non-zero Lie ideal $U$ of $K$ must contain $[K, K]_{\Gamma}$.

\section{References}

[1] W.E. Barnes :"On the gamma rings of Nobusawa”, Pacific J. Math 18 (1966) 411-422.

[2] W. Baxter :"Lie simplicity of a special class of associative rings". Proc. Amer. Math. Soc. 7 (1958), $855-863$.

[3] I.N.Herstein :" Topics in Ring Theory”, The University of Chicago Press, (1969).

[4] I.N.Herstein :"Lie and Jordan System in Simple Rings with Involution”, Amer. Journal Math. 78(1956), 629-649.

[5] L. Luh :"On the theory of simple Gamma rings," Michigan Math. J.,16(1969), 65-75.

[6] N.Nobusawa: "On a generalization of the ring theory" Osaka J. Math. 1(1964), 81-89.

[7] A.C.Paul and Sabur Uddin:"Lie and Jordan Structure in Simple Gamma Rings" Journal of Physical Sciences Vol.14 (2010), $77-86$.

[8] A.C.Paul and Sabur Uddin:"Lie Structure in Simple Gamma Rings" International Journal of Pure and Applied Sciences and Technology Vol.4(2) (2011), 63-70. 